\title{
Rapid Adaptation of Neural Machine Translation to New Languages
}

\author{
Graham Neubig, Junjie Hu \\ Language Technologies Institute, Carnegie Mellon University \\ \{gneubig, junjieh\}@cs.cmu.edu
}

\begin{abstract}
This paper examines the problem of adapting neural machine translation systems to new, low-resourced languages (LRLs) as effectively and rapidly as possible. We propose methods based on starting with massively multilingual "seed models", which can be trained ahead-of-time, and then continuing training on data related to the LRL. We contrast a number of strategies, leading to a novel, simple, yet effective method of "similar-language regularization", where we jointly train on both a LRL of interest and a similar high-resourced language to prevent over-fitting to small LRL data. Experiments demonstrate that massively multilingual models, even without any explicit adaptation, are surprisingly effective, achieving BLEU scores of up to 15.5 with no data from the LRL, and that the proposed similarlanguage regularization method improves over other adaptation methods by 1.7 BLEU points average over 4 LRL settings. ${ }^{1}$
\end{abstract}

\section{Introduction}

When disaster strikes, news and social media are invaluable sources of information, allowing humanitarian organizations to rapidly mitigate crisis situations and save lives (Vieweg et al., 2010; Neubig et al., 2011; Starbird et al., 2012). However, language barriers looms large over these efforts, especially when disasters occur in parts of the world that use less common languages. In these cases, machine translation (MT) technology can be a valuable tool, with one widely-heralded success story being the deployment of Haitian Creoleto-English translation systems during the earthquakes in Haiti (Lewis, 2010; Munro, 2010).

However, data-driven MT systems, particularly neural machine translation (NMT; Kalchbrenner

\footnotetext{
${ }^{1}$ Code to reproduce experiments at https://github. $\mathrm{com} /$ neubig/rapid-adaptation
}

and Blunsom (2013); Bahdanau et al. (2015)), require large amounts of training data, and creating high-quality systems in low-resource languages (LRLs) is a difficult challenge where research efforts have just begun (Gu et al., 2018). Another hurdle, which to our knowledge has not been covered in previous research, is the time it takes to create such a system. In a crisis situation, time is of the essence, and systems that require days or weeks of training will not be desirable or even feasible.

In this paper we focus on the question: how can we create MT systems for new language pairs as accurately as possible, and as quickly as possible? To examine this question we propose NMT methods at the intersection of cross-lingual transfer learning (Zoph et al., 2016) and multilingual training (Johnson et al., 2016), two paradigms that, to our knowledge, have not been used together in previous work. Our methods, laid out in $\S 2$ follow the process of training a seed model on a large number of languages, then fine-tuning the model to improve its performance on the language of interest. We propose a novel method of similar-language regularization (SLR) where training data from a second similar languages is used to help prevent over-fitting to the small LRL dataset.

In the experiments in $\S 3$, we attempt to answer two questions: (1) Which method of creating multilingual systems and adapting them to an LRL is the most effective way to increase accuracy? (2) How can we create the strongest system possible with a bare minimum of training time? The results are sometimes surprising - we first find that a single monolithic model trained on 57 languages can achieve BLEU scores as high as 15.5 with no training data in the new source language whatsoever. In addition, the proposed method starting with a universal model then fine-tuning with the SLR proves most effective, achieving gains of 1.7 
BLEU points averaged over several language pairs compared to previous methods adapting to only the LRL.

\section{Training Paradigms}

In this paper, we consider the setting where we have a source $L R L$ of interest, and we want to translate into English. ${ }^{2}$ All of our adaptation methods are based on first training on larger data including other languages, then fine-tuning the model to be specifically tailored to the LRL. We first discuss a few multilingual training paradigms from previous literature $(\S 2.1)$, then discuss our proposed adaptation methods ( $(2.2)$.

\subsection{Multilingual Modeling Methods}

We use three varieties of multilingual training:

Single-source modeling ("Sing.") is the first method, using only parallel data between the LRL of interest and English. This method is straightforward and the resulting model will be most highly tailored to the final test language pair, but the method also has the obvious disadvantage that training data is very sparse.

Bi-source modeling ("Bi') trains an MT system with two source languages: one LRL that we would like to translate from, and a second highly related high-resource language (HRL): the helper source language. ${ }^{3}$ This method is inspired by Johnson et al. (2016), who examine multilingual translation models to/from English and two highly related languages such as Spanish/Portuguese or Japanese/Korean. The advantage of this method is that it allows the LRL to learn from a highly similar helper, potentially increasing accuracy.

All-source modeling ("All") trains not only on a couple source languages, but instead creates a universal model on all of the languages that we have at our disposal. In our experiments ( $\S 3.1)$ this entails training systems on 58 source languages, to our knowledge the largest reported in NMT experiments. ${ }^{4}$ This paradigm allows us to train a single

\footnotetext{
${ }^{2}$ Translation into LRLs, is a challenging and interesting problem in it's own right, but beyond the scope of the paper.

"Related" could mean different things: typologically related or having high lexical overlap. In our experiments our LRLs are all selected to have an helper that is highly similar in both aspects, but choosing an appropriate helper when this is not the case is an interesting problem for future work.

${ }^{4}$ In contrast to $\mathrm{Gu}$ et al. (2018), who train on 10 languages. Malaviya et al. (2017); Tiedemann (2018) train NMT on over 1,000 languages, but only as a feature extractor for downstream tasks; MT accuracy itself is not evaluated.
}

model that has wide coverage of vocabulary and syntax of a large number of languages, but also has the drawback in that a single model must be able to express information about all the languages in the training set within its limited parameter budget. Thus, it is reasonable to expect that this model may achieve worse accuracy than a model created specifically to handle a particular source language.

In the following, we will consider adaptation methods that focus on tailoring a more general model (i.e. bi-source or universal) to a more specific model (i.e. single-source or bi-source).

\subsection{Adaptation to New Languages}

As noted in the introduction, there are two major requirements: the accuracy of the system is important and the training time required from when we learn of a need for translation to when we can first start producing adequate results. Throughout the discussion, we will compare various adaptation paradigms with respect to these two aspects.

\subsubsection{Adaptation by Fine-tuning}

Our first adaptation method, inspired by Zoph et al. (2016) is based on fine-tuning to the source language of interest. Within our experiments, we will test this setting, but also make two distinctions between the types of adaptation:

Seed Model Variety: Zoph et al. (2016) performed experiments taking a bilingual system trained on a different language (e.g. French) and adapting it to a new LRL (e.g. Uzbek). We can also take universal model and adapt it to the new language, a setting that we examine (to our knowledge, for the first time) in this work.

Warm vs. Cold Start: Another contrast is whether we have training data for the LRL of interest while training the original system, or whether we only receive training data after the original model has already been trained. We call the former warm start, and the latter cold start. Intuitively, we expect warm-start training to perform better, as having access to the LRL of interest during the training of the original model will ensure that it can handle the LRL to some extent. However, the cold-start scenario is also of interest: we may want to spend large amounts of time training a strong model, then quickly adapt to a new language that we have never seen before in our training data as data becomes available. For the cold-start models, we start with a model that is only trained on the HRL similar to the LRL $\left(\mathrm{Bi}^{-}\right)$, or a model trained 


\begin{tabular}{l|rrr||l|l} 
LRL & train & dev & test & HRL & train \\
\hline aze & $5.94 \mathrm{k}$ & 671 & 903 & tur & $182 \mathrm{k}$ \\
bel & $4.51 \mathrm{k}$ & 248 & 664 & rus & $208 \mathrm{k}$ \\
glg & $10.0 \mathrm{k}$ & 682 & 1,007 & por & $185 \mathrm{k}$ \\
slk & $61.5 \mathrm{k}$ & 2,271 & 2,445 & ces & $103 \mathrm{k}$
\end{tabular}

Table 1: Data sizes in sentences for LRL/HRL pairs

on all languages but the $\mathrm{LRL}\left(\mathrm{All}^{-}\right)$.

\subsubsection{Similar-Language Regularization}

One problem with adapting to a small amount of data in the target language is that it will be very easy for the model to over-fit to the small training set. To alleviate this problem, we propose a method of similar language regularization: while training to adapt to the language of interest, we also add some data from another similar HRL that has sufficient resources to help prevent overfitting. We do this in two ways:

Corpus Concatenation: Simply concatenate the data from the two corpora, so that we have a small amount of data in the LRL, and a large amount of data in the similar HRL.

Balanced Sampling: Every time we select a minibatch to do training, we either sample it from the LRL, or from the HRL according to a fixed ratio. We try different sampling strategies, including sampling with a 1-to-1 ratio, 1-to-2 ratio, and 1-to-4 ratio for the LRL and HRL respectively.

\section{Experiments}

\subsection{Experimental Setup}

We perform experiments on the 58-language-toEnglish TED corpus (Qi et al., 2018), which is ideal for our purposes because it has a wide variety of languages over several language families, some high-resourced and some low-resourced. Like Qi et al. (2018), we experiment with Azerbaijani (aze), Belarusian (bel), and Galician (glg) to English, and also additionally add Slovak (slk), a slightly higher resourced language, for contrast. These languages are all paired with a similar HRL: Turkish (tur), Russian (rus), Portuguese (por), and Czech (ces) respectively. Data sizes are shown in Table 1.

Models are implemented using xnmt (Neubig et al., 2018), commit 8173b1f, and start with the recipe for training on IWSLT TED ${ }^{5}$. The model consists of an attentional neural machine translation model (Bahdanau et al., 2015), using bi-directional LSTM encoders, 128-dimensional

\footnotetext{
${ }^{5}$ Found in examples/stanford-iwslt/
}

word embeddings, 512-dimensional hidden states, and a standard LSTM-based decoder.

Following standard practice (Sennrich et al., 2016; Denkowski and Neubig, 2017), we break low-frequency words into subwords using the sentencepiece toolkit. ${ }^{6}$ There are two alternatives for creating subword units: jointly learning subwords over all source language, or separately learning subwords for each source language, then taking the union of all the subword vocabularies as the vocabulary for the multilingual model. Previous work on multilingual training has preferred the former (Nguyen and Chiang, 2017), but in this paper we use the latter for two reasons: (1) because data in the LRL will not affect the subword units from the other languages, in the cold-start scenario we can postpone creation of subword units for the LRL until directly before we start training on the LRL itself, and (2) we need not be concerned with the LRL being "overwhelmed" by the higher-resourced languages when calculating statistics used in the creation of subword units, because all languages get an equal share. ${ }^{7}$ In the experiments, we use a subword vocabulary of 8,000 for each language.

We also compare with two additional baselines: phrase-based MT implemented in Moses, ${ }^{8}$ and unsupervised NMT implemented in undreamt. ${ }^{9}$ Moses is trained on the bilingual data only (training multilingually reduced average accuracy), and undreamt is trained on all monolingual data available for the LRL and English.

\subsection{Experimental Results}

Table 2 shows our main translation results, with warm-start scenarios in the upper half and coldstart scenarios in the lower half.

Does Multilingual Training Help? To answer this question, we can compare the warm-start Sing., Bi, and All settings, and find that the answer is a resounding yes, gains of 7-13 BLEU points are obtained by going from single-source to bi-source or all-source training, corroborating previous work (Gu et al., 2018). Bi-source models tend to perform slightly better than all-source models, indicating that given identical parameter

\footnotetext{
${ }^{6}$ https://github.com/google/ sentencepiece, using the unigram training setting.

${ }^{7}$ Preliminary experiments found both comparable: with scores of 20.1 and 19.4 for separate and joint respectively.

${ }^{8}$ http://statmt.org/moses

${ }^{9}$ https://github.com/artetxem/undreamt
} 


\begin{tabular}{|c|c|c|c|c|c|c|}
\hline & Strategy & aze/tur & bel/rus & glg/por & slk/ces & Avg. \\
\hline \multirow{2}{*}{\multicolumn{2}{|c|}{$\begin{array}{l}\text { Phrase-based } \\
\text { Unsupervised NMT }\end{array}$}} & (5.9 & 10.5 & 22.3 & 23.0 & 15.4 \\
\hline & & 0.0 & 0.3 & 0.4 & 0.0 & 0.2 \\
\hline \multirow{9}{*}{ 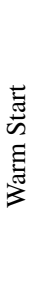 } & Sing. & 2.7 & 2.8 & 16.2 & 24.0 & 11.4 \\
\hline & $\mathrm{Bi}$ & 10.9 & 15.8 & 27.3 & 26.5 & 20.1 \\
\hline & All & 9.7 & 16.7 & 26.5 & 25.0 & 19.5 \\
\hline & $\mathrm{Bi} \rightarrow$ Sing. & 11.4 & 16.3 & 27.5 & 27.1 & 20.6 \\
\hline & All $\rightarrow$ Sing. & 10.1 & 17.5 & 28.2 & 27.4 & 20.8 \\
\hline & $\mathrm{All} \rightarrow \mathrm{Bi}$ & 11.7 & 18.3 & 28.8 & 28.2 & 21.8 \\
\hline & All $\rightarrow$ Bi 1-1 & 10.2 & 18.3 & 28.8 & 28.3 & 21.4 \\
\hline & All $\rightarrow$ Bi 1-2 & 11.0 & 17.5 & 29.1 & 28.2 & 21.4 \\
\hline & All $\rightarrow$ Bi 1-4 & 11.1 & 17.9 & 28.5 & 27.9 & 21.3 \\
\hline \multirow{8}{*}{ 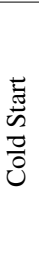 } & $\mathrm{Bi}^{-}$ & 3.8 & 2.5 & 8.6 & 5.4 & 5.1 \\
\hline & $\mathrm{All}^{-}$ & 3.7 & 3.5 & 15.5 & 7.3 & 7.5 \\
\hline & $\mathrm{Bi}^{-} \rightarrow$ Sing. & 8.7 & 11.8 & 25.4 & 26.8 & 18.2 \\
\hline & $\mathrm{All}^{-} \rightarrow$ Sing. & 8.8 & 15.3 & 26.5 & 27.6 & 19.5 \\
\hline & $\mathrm{All}^{-} \rightarrow \mathrm{Bi}$ & 10.7 & 17.4 & 28.4 & 28.0 & 21.2 \\
\hline & $\mathrm{All}^{-} \rightarrow \mathrm{Bi}$ 1-1 & 10.5 & 16.0 & 28.0 & 28.2 & 20.7 \\
\hline & $\mathrm{All}^{-} \rightarrow \mathrm{Bi} 1-2$ & 10.7 & 17.1 & 28.3 & 27.9 & 21.0 \\
\hline & $\mathrm{All}^{-} \rightarrow \mathrm{Bi}$ 1-4 & 11.0 & 17.4 & 28.4 & 27.6 & 21.1 \\
\hline
\end{tabular}

Table 2: BLEU for single-source (Sing.), bi-source (Bi), and all-source universal (All) models, with adapted counterparts. 1-1, 1-2, 1-4 indicate balanced sampling from $\S 2.2$. Bold indicates highest score.

capacity, training on a highly resourced language is effective. Comparing with the phrase-based baseline, as noted by Koehn and Knowles (2017) NMT tends to underperform on low-resource settings when trained only on the data available for these languages. However, multilingual training of any variety quickly remedies this issue; all outperform phrase-based handily.

More interestingly, examining the cold-start results, we can see that even systems with no data in the target language are able to achieve nontrivial accuracies, up to $15.5 \mathrm{BLEU}$ on glg-eng. Interestingly, in the cold-start scenario, the $\mathrm{All}^{-}$ model bests the $\mathrm{Bi}^{-}$model, indicating that massively multilingual training is more useful in this setting. In contrast, the unsupervised NMT model struggles, achieving a BLEU score of around 0 for all language pairs - this is because unsupervised NMT requires high-quality monolingual embeddings from the same distribution, which can be trained easily in English, but are not available in the low-resource languages we are considering.

Does Adaptation Help? Regarding adaptation, we can first observe that regardless of the original model and method for adaptation, adaptation is helpful, particularly (and unsurprisingly) in the cold-start case. When adapting directly to only the target language (" $\rightarrow$ Sing."), adapting from the massively multilingual model performs better, indicating that information about all input languages is better than just a single language. Next, comparing with our proposed method of adding similar
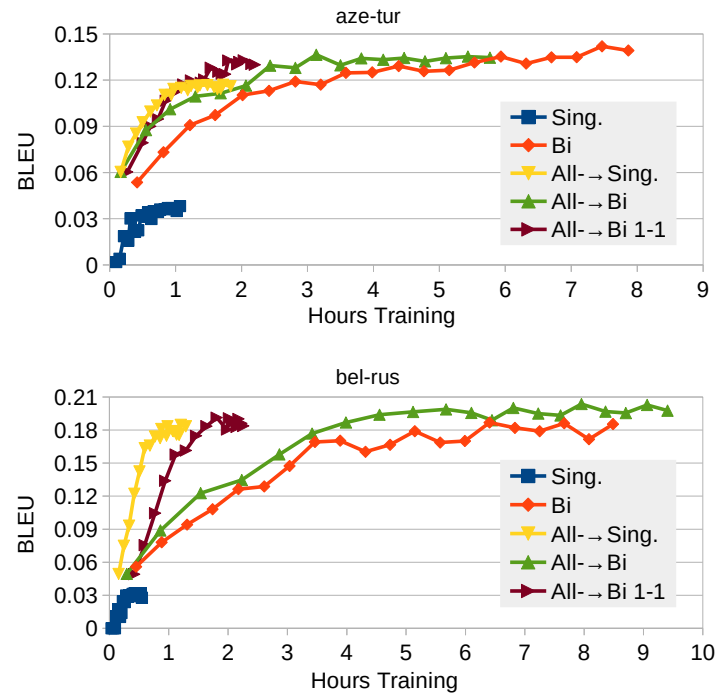

Figure 1: Example of adaptation on the aze-eng and bel-eng development sets

language regularization (" $\rightarrow$ Bi"), we can see that this helps significantly over adapting directly to the LRL, particularly in the cold-start case where we can observe gains of up to 1.7 BLEU points. Finally, in our data setting, corpus concatenation outperforms balanced sampling in both the coldstart and warm-start scenarios.

How Can We Adapt Most Efficiently? Finally, we revisit adapting to new languages efficiently, with Figure 1 showing BLEU vs. hours training for the aze/tur and bel/rus source language pairs (others were similar). We can see that in all cases the cold-start models (All ${ }^{-} \rightarrow$ ) either outperform or are comparable in final accuracy to the fromscratch single-source and bi-source models. In addition, all of the adapted models converge faster than the bi-source from-scratch trained models, indicating that adapting from seed models is a good strategy for rapid construction of MT systems in new languages. Comparing the cold-start adaptation strategies, we can see that in general, the higher the density of target language training data, the faster the training converges to a solution, but the worse the final solution is. This suggests that there is a speed/accuracy tradeoff in the amount of similar language regularization we apply during fine-tuning.

\section{Related Work}

While adapting MT systems to new languages is a long-standing challenge (Schultz and Black, 2006; Jabaian et al., 2013), multilingual NMT is highly promising in its ability to abstract across 
language boundaries (Firat et al., 2016; Ha et al., 2016; Johnson et al., 2016). Results on multilingual training for low-resource translation $(\mathrm{Gu}$ et al., 2018; Qi et al., 2018) further demonstrates this potential, although these works do not consider adaptation to new languages, the main focus of our work. Notably, we did not examine partial freezing of parameters, another method proven useful for cross-lingual adaptation (Zoph et al., 2016); this is orthogonal to our multi-lingual training approach but the two methods could potentially be combined. Finally, unsupervised NMT approaches (Artetxe et al., 2017; Lample et al., 2018 , 2017) require no parallel data, but rest on strong assumptions about high-quality comparable monolingual data. As we show, when this assumption breaks down these methods fail to function, while our cold-start methods achieve non-trivial accuracies even with no monolingual data.

\section{Conclusion}

This paper examined methods to rapidly adapt MT systems to new languages by fine-tuning. In both warm-start and cold-start scenarios, the best results were obtained by adapting a pre-trained universal model to the low-resource language while regularizing with similar languages.

\section{Acknowledgements}

The authors thank Jaime Carbonell, Xinyi Wang, Rebecca Knowles, Arya McCarthy, and anonymous reviewers for their constructive comments on this paper.

This work is sponsored by Defense Advanced Research Projects Agency Information Innovation Office (I2O). Program: Low Resource Languages for Emergent Incidents (LORELEI). Issued by DARPA/I2O under Contract No. HR0011-15C0114. The views and conclusions contained in this document are those of the authors and should not be interpreted as representing the official policies, either expressed or implied, of the U.S. Government. The U.S. Government is authorized to reproduce and distribute reprints for Government purposes notwithstanding any copyright notation here on.

\section{References}

Mikel Artetxe, Gorka Labaka, Eneko Agirre, and Kyunghyun Cho. 2017. Unsupervised neural machine translation. arXiv preprint arXiv:1710.11041.
Dzmitry Bahdanau, Kyunghyun Cho, and Yoshua Bengio. 2015. Neural machine translation by jointly learning to align and translate. In Proc. ICLR.

Michael Denkowski and Graham Neubig. 2017. Stronger baselines for trustable results in neural machine translation. In Proc. WNMT.

Orhan Firat, Kyunghyun Cho, and Yoshua Bengio. 2016. Multi-way, multilingual neural machine translation with a shared attention mechanism. In Proc. NAACL, pages 866-875.

Jiatao Gu, Hany Hassan, Jacob Devlin, and Victor OK Li. 2018. Universal neural machine translation for extremely low resource languages. Proc. NAACL.

Thanh-Le Ha, Jan Niehues, and Alexander Waibel. 2016. Toward multilingual neural machine translation with universal encoder and decoder. arXiv preprint arXiv:1611.04798.

Bassam Jabaian, Laurent Besacier, and Fabrice Lefevre. 2013. Comparison and combination of lightly supervised approaches for language portability of a spoken language understanding system. IEEE Transactions on Audio, Speech, and Language Processing, 21(3):636-648.

Melvin Johnson et al. 2016. Google's multilingual neural machine translation system: Enabling zero-shot translation. TACL.

Nal Kalchbrenner and Phil Blunsom. 2013. Recurrent continuous translation models. In Proc. EMNLP, pages 1700-1709.

Philipp Koehn and Rebecca Knowles. 2017. Six challenges for neural machine translation. Proc. WNMT.

Guillaume Lample, Ludovic Denoyer, and Marc'Aurelio Ranzato. $2017 . \quad$ Unsupervised machine translation using monolingual corpora only.

Guillaume Lample, Myle Ott, Alexis Conneau, Ludovic Denoyer, and Marc'Aurelio Ranzato. 2018. Phrase-based \& neural unsupervised machine translation. arXiv preprint arXiv:1804.07755.

William D. Lewis. 2010. Haitian Creole: how to build and ship an MT engine from scratch in 4 days, 17 hours, \& 30 minutes. In Proc. EAMT.

Chaitanya Malaviya, Graham Neubig, and Patrick Littell. 2017. Learning language representations for typology prediction. In Proc. EMNLP.

Robert Munro. 2010. Crowdsourced translation for emergency response in Haiti: the global collaboration of local knowledge. In Proc. AMTA Workshop on Collaborative Crowdsourcing for Translation.

Graham Neubig, Yuichiroh Matsubayashi, Masato Hagiwara, and Koji Murakami. 2011. Safety information mining - what can NLP do in a disaster -. In Proc. IJCNLP, pages 965-973. 
Graham Neubig, Matthias Sperber, Xinyi Wang, Matthieu Felix, Austin Matthews, Sarguna Padmanabhan, Ye Qi, Devendra Singh Sachan, Philip Arthur, Pierre Godard, John Hewitt, Rachid Riad, and Liming Wang. 2018. XNMT: The extensible neural machine translation toolkit. In Proc. AMTA, Boston.

Toan Q. Nguyen and David Chiang. 2017. Transfer learning across low-resource, related languages for neural machine translation. pages 296-301.

Ye Qi, Devendra Sachan, Matthieu Felix, Sarguna Padmanabhan, and Graham Neubig. 2018. When and why are pre-trained word embeddings useful for neural machine translation? In Proc. NAACL, New Orleans, USA.

Tanja Schultz and Alan W Black. 2006. Challenges with rapid adaptation of speech translation systems to new language pairs. In Proc. ICASSP, volume 5. IEEE.

Rico Sennrich, Barry Haddow, and Alexandra Birch. 2016. Neural machine translation of rare words with subword units. In Proc. ACL, pages 1715-1725.

Kate Starbird, Grace Muzny, and Leysia Palen. 2012. Learning from the crowd: Collaborative filtering techniques for identifying on-the-ground Twitterers during mass disruptions. In Proc. ISCRAM.

Jörg Tiedemann. 2018. Emerging language spaces learned from massively multilingual corpora. arXiv preprint arXiv:1802.00273.

Sarah Vieweg, Amanda L Hughes, Kate Starbird, and Leysia Palen. 2010. Microblogging during two natural hazards events: what Twitter may contribute to situational awareness. In Proc. CHI, pages 10791088 .

Barret Zoph, Deniz Yuret, Jonathan May, and Kevin Knight. 2016. Transfer learning for low-resource neural machine translation. In Proc. EMNLP, pages $1568-1575$. 